\title{
A Meta-analysis of the External Treatment of Traditional Chinese Medicine for Granulomatous Mastitis
}

\author{
Liang Hongyi ${ }^{1}$, Chen Hanhan ${ }^{2, *}$ \\ ${ }^{1}$ College of Traditional Chinese Medicine, Shandong University of Traditional Chinese Medicine, Jinan, China \\ ${ }^{2}$ Department of Breast and Thyroid Surgery, Affiliated Hospital of Shandong University of Traditional Chinese Medicine, Jinan, China
}

\section{Email address:}

827491136@qq.com (Liang Hongyi), szychenhan@163.com (Chen Hanhan)

${ }^{*}$ Corresponding author

\section{To cite this article:}

Liang Hongyi, Chen Hanhan. A Meta-analysis of the External Treatment of Traditional Chinese Medicine for Granulomatous Mastitis. Science Discovery. Vol. 9, No. 1, 2021, pp. 21-26. doi: 10.11648/j.sd.20210901.14

Received: February 2, 2021; Accepted: March 3, 2021; Published: March 9, 2021

\begin{abstract}
Objective: to systematically evaluate the clinical efficacy of external treatment of granulomatous mastitis (including clinical efficiency and the change of mass size). Methods: the computer was used to search CNKI, Wanfang database, VIP net, CBM, PubMed and Embase, and it was used to search and select the clinical randomized controlled trials of treating granulomatous mastitis by external treatment of traditional Chinese medicine, the retrieval time limit was from the establishment of the database to December 2020, and the key words were granulomatous mastitis, external treatment of traditional Chinese medicine. After selecting literatures, extracting datas and evaluating the risk of bias included in the study, the data included in the study were analyzed by RevMan5.3 software. Results: Seven clinical randomized controlled trials were included and 600 patients met the inclusion criteria. Seven of the trials were effective observations, and four of them were tumor size observations. There were 310 cases in the experimental group and 290 cases in the control group. All the included documents were Chinese literature. The results of the study showed that the effective rate of the experimental group (combined with the external treatment of traditional Chinese medicine) for granulomatous mastitis was $95.9 \%$, which was significantly higher than that of the control group (without the external treatment of traditional Chinese medicine). The effective rate was $82.8 \%$ [95\% CI (1.02, 1.22), $\mathrm{Z}=2.41, \mathrm{P}=0.02<0.05]$. The experimental group (combined with the external treatment of traditional Chinese medicine) had a significantly higher degree of the tumor size recovery than the control group (without the external treatment of traditional Chinese medicine) [95\% CI $(-2.05,-1.83), Z=34.91, \mathrm{P}<0.00001]$. Conclusion: the external treatment of granulomatous mastitis had a good clinical effect. It not only improved the clinical efficiency, but also effectively reduced the size of breast mass, which was worthy of clinical promotion. However, due to the low quality of clinical trials, further verification was needed.
\end{abstract}

Keywords: External Treatment of Traditional Chinese Medicine, Granulomatous Mastitis, Meta Analysis, Effective Rate, The Size of Breast Mass

\section{中医外治治疗肉芽肿性乳腺炎的荟萃分析}

\author{
梁鸿艺 ${ }^{1}$, 陈翰翰 ${ }^{2 *}$ \\ ${ }^{1}$ 山东中医药大学中医学院, 济南, 中国 \\ ${ }^{2}$ 山东中医药大学附属医院乳腺甲状腺外科, 济南, 中国 \\ 邮箱 \\ 827491136@qq.com (梁鸿艺), szychenhan@163.com（陈翰翰）
}

摘要: 目的: 系统评价中医外治治疗肉芽肿性乳腺炎的治疗效果。方法: 计算机检索中国知网、万方数据库、维普网、 CBM、PubMed、Embase，检索篎选出中医外治治疗肉芽肿性乳腺炎的临床随机对照试验，检索时间限制为自建库始 
至2020年12月, 检索关键词为肉芽肿性乳腺炎、中医外治, 笁选文献、提取资料并对纳入研究的偏倚风险进行评价。 荟萃分析采用RevMan5.3软件对纳入研究的数据进行。结果: 7篇临床随机对照试验文献, 600名患者符合纳入标准, 7 项为有效率观察, 4项为肿块大小观察, 实验组 310 例, 对照组290例, 所有纳入的文献均为中文文献。研究结果显示, 实验组治疗肉芽肿性乳腺炎的有效率为 $95.9 \%$, 明显高于对照组有效率为 $82.8 \%[95 \% \mathrm{CI}(1.02,1.22), \mathrm{Z}=2.41, \mathrm{P}=0.02<0.05]$; 实验组肿块大小恢复程度明显高于对照组 $[95 \% \mathrm{CI}(-2.05,-1.83), \mathrm{Z}=34.91, \mathrm{P}<0.00001]$ 。结论：中医外治医治肉芽肿 性乳腺炎的临床疗效较好, 不仅可以提高临床有效率, 而且可以有效缩小肿块大小, 值得临床推广和使用。但由于临 床试验质量较低, 需要做进一步的验证。

关键词: 中医外治, 肉芽肿性乳腺炎, Meta分析, 有效率, 肿块大小

\section{1. 引言}

肉芽肿性乳腺炎（GLM），又被称为特发性肉芽肿性 乳腺炎(IGM) [1], 是一种以乳腺组织中出现肉芽肿为主要 病理表现的炎症性乳腺慢性疾病。女性的乳腺小叶是主要 的侵犯部位, 由此获得肉芽肿性小叶性乳腺炎的称号 [2]。 最早于1972年由Kessler[3]等人提出，常见于育龄期女性 [4]。GLM归属于中医学的“乳痛”范畴, 目前临床治疗的 手段有多种治疗方式，而使用较多的是手术治疗和保守治 疗两种 [5]。随着人们对生活质量的追求逐步增加, 手术治 疗创口大，影响乳房美观性; 保守治疗中激素治疗副作用 多, 影响身体恢复[6]。寻找一种有效而又较大限度维持乳 房美观度的治疗方法符合临床的需要[7],近年来已有多项 临床研究表明中医外治法能够治疗肉芽肿性乳腺炎, 但尚 缺乏临床有效率、肿块大小变化的系统评价。本研究纳入 了截至目前国内外公开发表的中医外治法治疗肉芽肿性 乳腺炎的随机临床对照试验 (RCT), 对纳入研究的试验 进一步荟萃分析, 循证依据和指导经验也进一步获得。

\section{2. 研究资料与方法}

\section{1. 文献纳入标准}

\subsection{1. 纳入类型}

文献必须是临床随机对照试验; 实验组干预措施为化 腐、引流、外敷、塌渍、火罐等中医外治疗法甚至联合其 他方式疗法治疗, 对照组干预措施为其他方式疗法干预或 者不进行干预; 文献语种仅限于中文和英文。

\subsection{2. 试验对象}

纳入患者的诊断需要根据《现代中医乳房病学》[8] 拟定的评价标准。

\section{2. 文献排除标准}

(1)文献中实验设计统计方法不符且设计不严谨; (2)重 复发表的文献。

\section{3. 文献检索策略}

检索数据库中文数据库以中国知网、万方数据库、 CBM和维普网为代表, 英文数据库以PubMed和Embase为 代表, 从建立数据库至 2020 年 5 月被作为检索期限, 各个 数据库分别检索并注意合并, 避免遗检。以“肉芽肿性乳 腺炎”为检索关键词, 二次检索“中医外治”, 再筛选出有 “随机分组”的文献, 英文检索词为 granulomatous mastitis、 external treatment of traditional Chinese medicine等。

\section{4. 文献资料提取与质量评价}

文献的纳入标准和排除标准被严格遵守, 两名研究人 员单独进行篎选, 获得合乎标准的文献并提取资料, 对其 进行质量评价, 并用Note Express进行篮选和排除重复。 提取的资料需要包括作者、文章发表时间、地域、样本总 量、分组情况、干预措施、结局指标并进行评分。

\section{5. 统计学分析}

采用RevMan5.3软件对所收集的数据进行统计学分析。 标准化均数差 $(M D)$ 和 $95 \%$ 可信区间 $(C I)$ 作为连续型 变量资料的研究指标, 此外相对危险度 $(R R)$ 及 $95 \%$ 可信 区间 $(C I)$ 便作为二分类变量研究资料的效应指标。异质 性检验采用 $P$ 值进行表示。如果 $P>0.05$, 表示异质性无统 计学意义, 采用固定效应模型; 如果 $P \leq 0.05$, 表示异质性 有统计学意义, 采用随机效应模型。亚组分析和敏感性分 析可以应用于异质性过大时探索异质性可能的来源。

\section{3. 结果}

\section{1. 文献检索结果}

计算机检索各大数据库中有关中医外治法以及其他 疗法治疗肉芽肿性乳腺炎的相关文献, 共 280 篇, 进一步 篮查后获得RCT 7篇, 文献篮查步骤见下图1, 文献纳入信 息见下表1。 


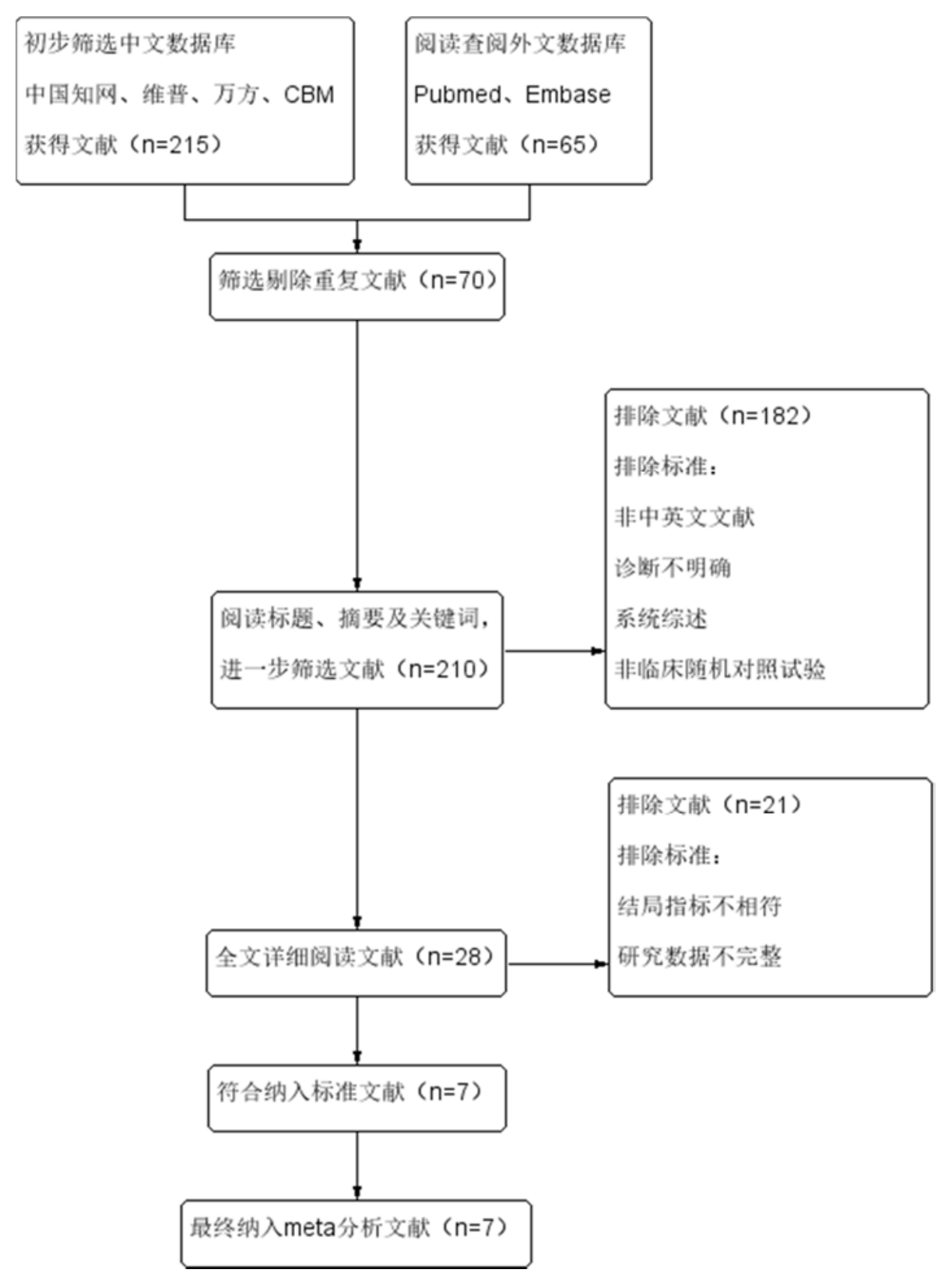

图1 流程图。

表1 文献纳入信息表。

\begin{tabular}{|c|c|c|c|c|c|c|}
\hline \multirow{2}{*}{ 第一作者 } & \multirow{2}{*}{$\begin{array}{l}\text { 发表 } \\
\text { 年份 }\end{array}$} & \multicolumn{2}{|c|}{ 样本量 (例) } & \multicolumn{2}{|l|}{ 干预方式 } & \multirow{2}{*}{ 观察指标 } \\
\hline & & 实验组 & 对照组 & 实验组 & 对照组 & \\
\hline 关青青[9] & 2018 & 30 & 30 & $\begin{array}{l}\text { 拔毒生肌散合溃疡油换药+中药“非哺乳 } \\
\text { 期乳腺炎二号方”口服 }\end{array}$ & $\begin{array}{l}\text { 银离子抗菌敷料换药+中药“非哺乳乳 } \\
\text { 腺炎二号方”口服期 }\end{array}$ & 有效率、肿块大小 \\
\hline 张娟 [10] & 2019 & 30 & 30 & 阳和膏外用+曲安奈德乳管冲洗 & 曲安奈德乳管冲洗 & 有效率、肿块大小 \\
\hline 李玉洁[11] & 2016 & 32 & 32 & 中医“提脓祛腐”综合外治+手术治疗组 & 手术治疗组 & 有效率 \\
\hline 杨乐平[12] & 2017 & 40 & 20 & 中医外治组 & 糖皮质激素局部封闭治疗组 & 有效率、肿块大小 \\
\hline 王灿[13] & 2019 & 108 & 108 & 局部注射曲安奈德+中药塌渍 & 局部注射曲安奈德 & 有效率 \\
\hline 蓝树英[14] & 2020 & 30 & 30 & 自血疗法+甲泼尼龙片 & 甲泼尼龙片 & 有效率 \\
\hline 装张利[15] & 2017 & 40 & 40 & 阳和汤+平衡火罐治疗 & 阳和汤 & 有效率、肿块大小 \\
\hline
\end{tabular}

\section{2. 纳入文献的质量评价}

共纳入7篇RCT研究, 共计肉芽肿性乳腺炎患者600名, 其中对照组290例, 实验组310例。现从以下七个方面对其 进行质量评价分析，具体情况见以下图2、图3。 


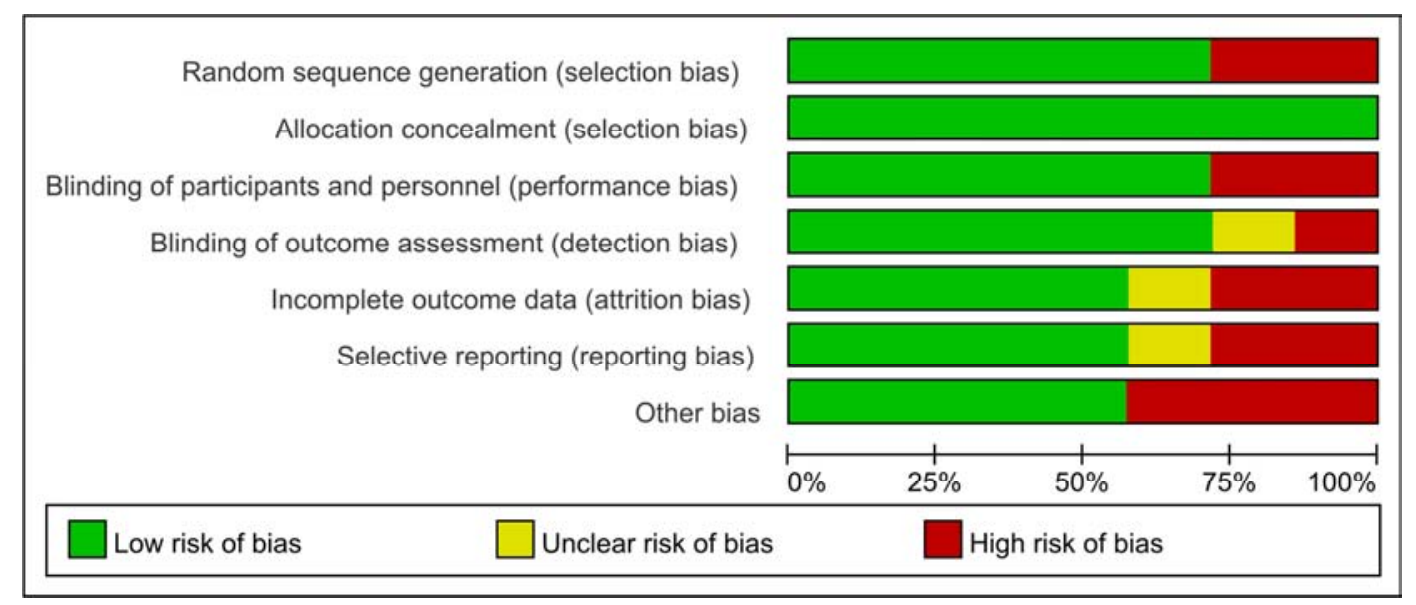

图2 偏倚风险分析图。

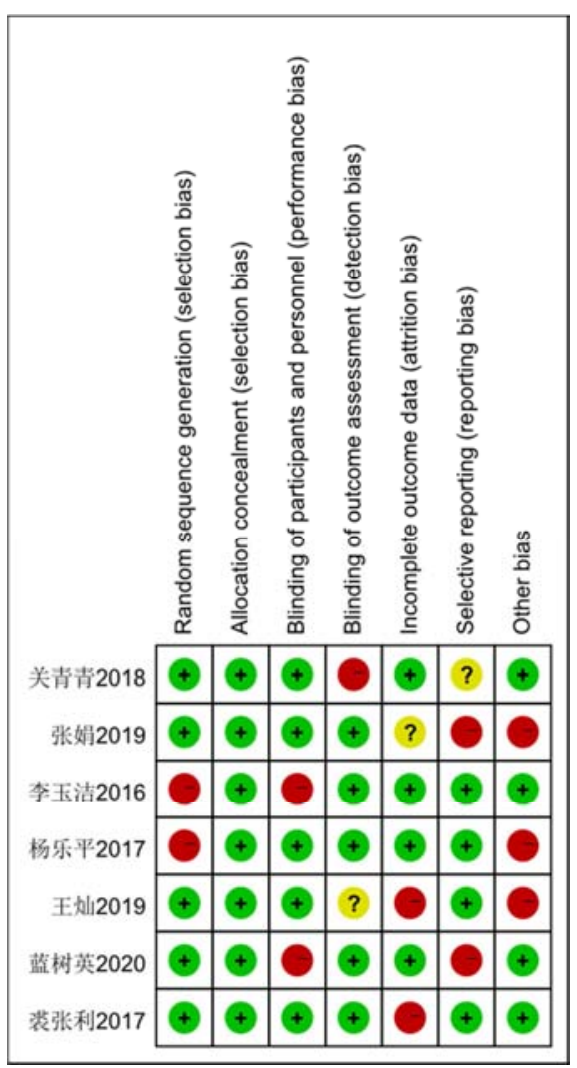

\section{3. 纳入研究资料的统计学分析}

\subsection{1. 指标 1 : 肿块大小}

该类型资料为连续型变量, 此类型资料的表达形式用 每个研究的均数和标准差来表示, 由于每个研究的结果变 量的量纲相同（乳房肿块最大直径、病灶最大径均用来表 示肿块大小），因此直接采用加权均数差表示效应指标。 共有4项研究涉及肿块大小这一指标, 对照组 120 例, 实验 组 140 例。进行异质性检验得知, $I^{2}=0 \%<50 \%, P=0.54>0.05$, 即不能认为研究间存在异质性, 采用固定效应模型。分析 结果揭示实验组对于乳房肿块大小的恢复程度优于对照 组, 二者相比差异具有统计学意义 $[95 \% C I(-2.05,-1.83)$, $Z=34.91, P<0.00001]$, 即认为中医外治法与其他方法在治 疗乳房肿块大小方面的差异, 中医外治法对乳房肿块大小 的恢复可以起促进作用。

在发表偏倚方面, 漏斗图是一种主观判断发表偏倚的 手段, 选择合适的横纵坐标对于分析发表偏倚至关重要 [16]。由图可知, 效应量为横坐标, 标准误为纵坐标, 3 项处于漏斗图的中上方, 1项处于漏斗图的下方, 所有数 据呈现向中心聚集的现象, 两侧基本对称, 图形呈现类似 倒置漏斗形状, 说明发表偏倚存在且可能偏倚较小。

下面两图（图4、图5) 是指标1采用Revman5.3中Meta 分析结果与漏斗图分析结果。

图3 偏倚风险总结图。

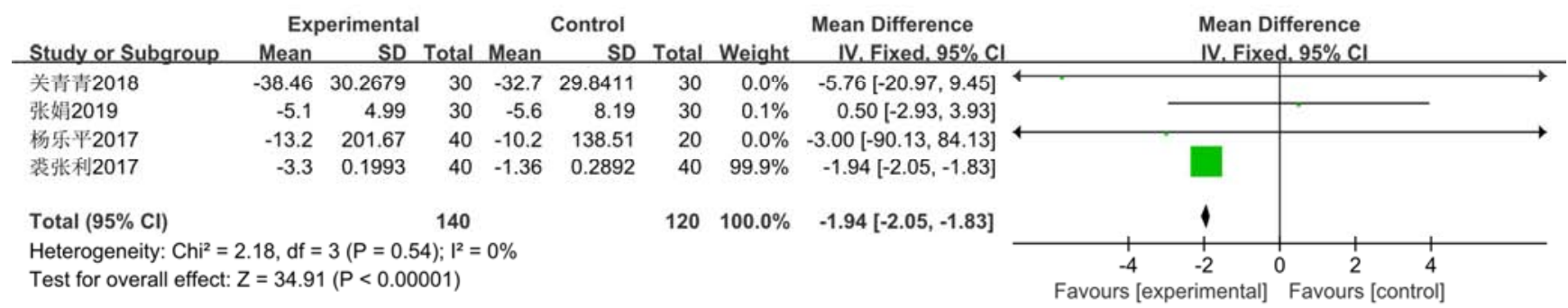

图4 肿块大小森林图。 


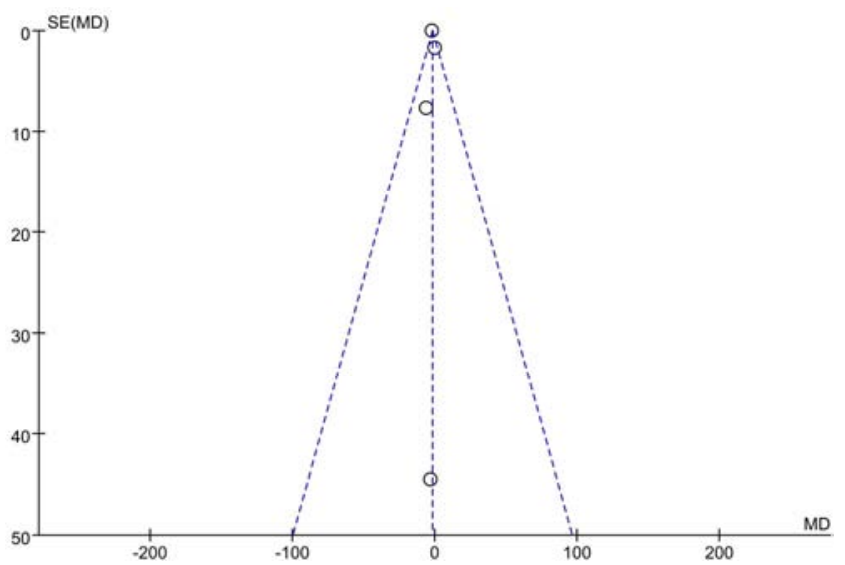

图5 肿块大小漏斗图。

\subsection{2. 指标2: 有效率}

该类型资料为二分类变量, 此类型资料每一个研究都 可以整理成一个四格表形式, 因此其效应指标可以是优势
比 $O R$ 、相对危险度 $R R$ 或者危险度差值 $R D$ 。此处以 $R R$ 为 效应指标进行统计学分析。共有7项研究涉及有效率这一 指标, 对照组 290 例, 实验组 310 例。进行异质性检验分析 得知, $I^{2}=71 \%>50 \%, P=0.002<0.05$, 即认为研究间存在异 质性, 故采用随机效应模型。分析结果表明, 实验组治疗 肉芽肿性乳腺炎的有效率为 $95.9 \%$, 明显高于对照组（有 效率为 $82.8 \%)$, 差异具有统计学意义 $[95 \% C I(1.02,1.22)$, $Z=2.41, P=0.02<0.05]$, 即认为中医外治法与其他方法治 疗肉芽肿性乳腺炎的有效率存在差异, 中医外治法对治疗 此病有促进作用。

在发表偏倚方面, 所有数据呈现向中间聚集的现象, 且两边基本呈对称分布, 基本表现为倒置漏斗的形状, 同 样说明发表偏倚可能存在但较小。

下面两图 (图6、图7) 即指标2采用Revman5.3中Meta 分析结果和漏斗图分析结果。

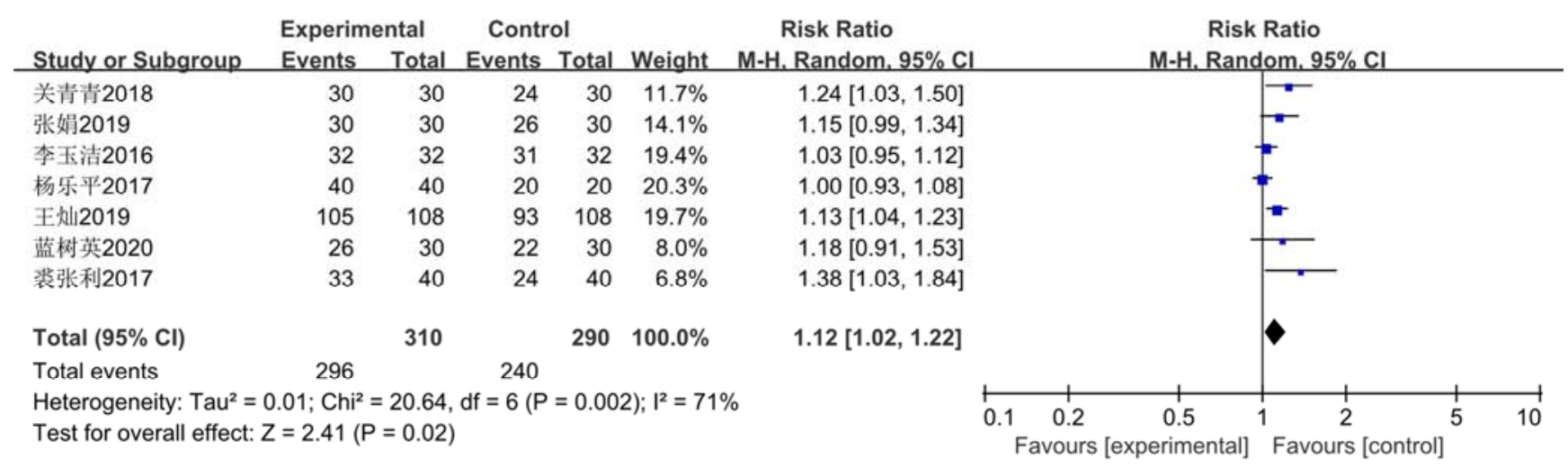

图6 有效率森林图。

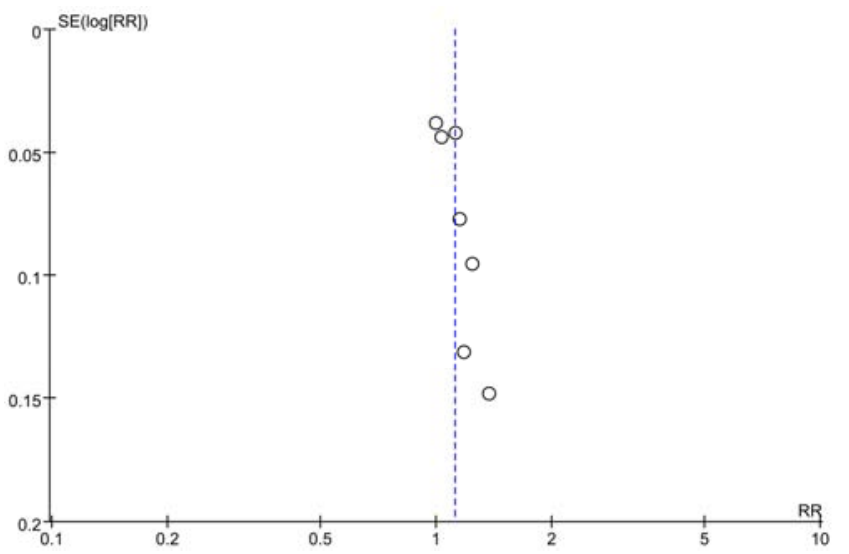

图7 有效率漏斗图。

\section{4. 讨论}

目前, 在临床上, 在西医治疗以及中医内治的基础之 上, 中医外治结合治疗肉芽肿性乳腺炎发挥越来越重要的 作用, Li S[17]等人认为导管灌洗和乳房按摩都是治疗肉 芽肿性乳腺炎的保守治疗方法, 副作用小而且危害小, 值 得提倡。王志华 $[18]$ 认为肉芽肿性乳腺炎发病与中医风邪
有关, 提倡在中医内治风药治疗的基础上运用洞室引流联 合社腐骚刮术这种有中医外治特色的方法进行治疗, 有效 地缩小了创口, 增强了美观性。胡金辉[19]、林毅[20]认 为肉芽肿性乳腺炎发病与脾胃有关, 分别提倡使用搔刮填 塞法与火针烙口治疗、提脓药捻引流法等多种中医外治法 社腐生肌, 从而有效地化瘀散结治疗肿块 [21]。简言之, 中医外治法治疗肉芽肿性乳腺炎在临床应用上发挥着不 可磨灭的作用, 临床上可以结合使用中医外治法, 发挥中 医疗法的特殊魅力。

通过以上质量评价与统计学分析, 共纳入研究7项, 患者共600例, 其中纳入研究的两个结局指标分别是肿块 大小和治疗有效率, 肿块大小共纳入患者 260 例, 治疗有 效率共纳入患者 600 例, 研究发现, 实验组总有效率 $95.5 \%$, 对照组总有效率 $82.8 \%$, 中医外治结合治疗组有效率较高 $(\mathrm{P}<0.05)$, 表示中医外治法参与肉芽肿性乳腺炎的治 疗可以提高治疗的有效率, 而且研究显示, 同等条件下实 验组肿块的缩小程度明显优于对照组, 表示中医外治法结 合治疗还能够促进肿块的缩小, 具有积极的促进作用 [22]。

但是, 此次研究还存在诸多不足与局限性, 样本含量 不够大不具有说服力; 检索到的文献均为中文文献, 缺少 符合纳入条件的国际研究, 涵盖范围不够广; 纳入研究的 
病例由于年龄、不同阶段等差异导致的偏倚未进行进一步 分析和研究; 所选取的效应指标不够多, 不具有很强的证 明能力等。因此, 希望能在以后的文献查阅以及病例纳入 中更加详细, 在数据分析与统计中更加严谨, 从而更好地 提高文献纳入水平与文章质量, 为临床提供更好的借鉴经 验。

\section{5. 结论}

中医外治治疗肉芽肿性乳腺炎的临床疗效较好, 不仅 可以有效地提高临床有效率, 而且可以进一步缩小肿块大 小, 值得临床推广和使用。但是由于临床试验质量较低, 仍需要做进一步的验证与研究。

\section{致谢}

感谢山东省中医药科技发展计划项目(2019-0159)的 赞助与支持。

\section{参考文献}

[1] Freeman CM, Lewis JD, Lower EE, et al. Refractory granulomas of breast: Benign or malignant disease[J]. Journal of Clinical Oncology, 2014, 32(26).

[2] 陈向红,赖俊辉,黄清春.肉芽肿性乳腺炎合并类风湿关节炎 1例 $[J]$.实用医学杂志,2018,(12):2094.

[3] Kessler E, Wolloeh Y. Granulomatous mastiffs: a lesion clinically simulating carcinoma[J]. Am J Clin Pathol, 1972, 58(6): 642-646.

[4] Goulabchand R, Hafidi A, Van de Perre P, et al. Mastitis in Autoimmune Diseases: Review of the Literature, Diagnostic Pathway, and Pathophysiological Key Players[J]. J Clin Med, 2020, 9(4).

[5] 薛田,曾一.曾一教授治疗肉芽肿性乳腺炎经验总结[J].四川 中医,2017,35(11):18-21.

[6] 中华预防医学会妇女保健分会乳腺保健与乳腺疾病防治学 组. 非哺乳期乳腺炎诊治专家共识 [J]. 中华乳腺病杂 志,2016,36(7):755-758.

[7] 朱华宇,司徒红林,关若丹,等.林毅运用中医特色疗法治疗肉 芽肿性乳腺炎经验举要[J].新中医,2013,45(6):189-191.
[8] 林毅, 唐汉钧.现代中医乳房病学 [M]. 北京: 人民卫生出版 社,2003.

[9] 关青青,刘守尧,韩静,等.祛腐生肌法治疗非哺乳期乳腺炎溃 后期临床研究[J].世界中医药,2018,13(06):1388-1391.

[10] 张娟.阳和膏外用联合曲安奈德乳管冲洗对肿块期肉芽肿 性乳腺炎的临床疗效观察[D]. 北京中医药大学,2019.

[11] 李玉洁.中医综合外治法治疗肉芽肿性小叶性乳腺炎前瞻 性临床研究[D].广州中医药大学,2016.

[12] 杨乐平,胡望,李伟佐,等.中医外治与激素封闭治疗肉芽肿性 乳腺炎的超声与临床疗效比较 [J]. 现代中西医结合杂 志,2017,26(23):2546-2548.

[13] 王灿. 中药塌渍在肉芽肿性乳腺炎中的作用 [J]. 智慧健 康,2019,5(36):98-99.

[14] 蓝树英,郭智涛.自血疗法联合甲泼尼龙片治疗肉芽肿性乳 腺炎的疗效观察 [J]. 广州中医药大学学 报,2020,37(01):68-73.

[15] 装张利,陈晓洁,吕晓皑,等.平衡火罐联合阳和汤治疗肉芽肿 性乳腺炎疗效观察 $[\mathrm{J}]$. 上海针尒杂 志,2017,36(09):1078-1081.

[16] 贾书冰,赵明沂,梁露花,等.漏斗图在Meta分析中的正确使用 研究 [J].数理医药学杂志,2013,26(4):402-405.

[17] Li S, Chen K, Zhu L, et al. Efficacy and safety of ductal lavage for non-lactational idiopathic granulomatous mastitis: A retrospective case series study[J]. Annals of Surgical Oncology, 2017, 24(2): 71-72.

[18] 李玲,王志华,马旭,等.王志华教授治疗肉芽肿性乳腺炎经验 总结[J].亚太传统医药,2020,16(01):106-107.

[19] 贺佳,胡金辉,杨杰,等.胡金辉运用益气健脾法治疗肉芽肿性 小叶性乳腺炎举要[J].中医药临床杂志,2020,32(01):27-30.

[20] 谢宛君, 林毅,钟少文,等. 基于数据挖掘的林毅教授治疗肉芽 肿性乳腺炎用药规律探析 [J]. 中国医药导 报,2019,16(3):120-123.

[21] 钟少文.林毅中医外治法治疗肉芽肿性乳腺炎 [J].河北中 医,2012,34(8):1128-1129.

[22] 陈一东,王伦保. 肉芽肿性乳腺炎治疗方法与临床经验分析 [J].中国处方药,2017,15(09):137-138. 